\title{
Pobreza y personas en situación de calle: Una nota sobre la experiencia internacional y lecciones para Chile
}

\author{
Poverty and homelessness: International \\ experience and lessons for Chile
}

\section{IGNACIO IRARRÁZAVAL}

Ignacio Irarrázaval es doctor en Política Social de la London School of Economics, actualmente es el director de Asuntos Públicos de la Universidad Católica de Chile. Dirección postal Avenida Libertador General Bernardo O’Higgins 340; iirarraz@uc.cl

\begin{abstract}
Resumen
Esta nota entrega algunos antecedentes sobre la experiencia internacional en el tema personas en situación de calle y la pobreza, e identifica algunas estrategias para la realidad chilena. La primera sección analiza algunas cuestiones conceptuales y metodológicas sobre la magnitud y la incidencia de personas en situación de calle en el contexto internacional. La segunda sección describe el perfil de este grupo social, en el contexto de los paises desarrollados, haciendo un breve paralelo con la situación en Chile. Por último, la tercera sección examina algunas de las estrategias que se han utilizado en Estados Unidos y, nuevamente, trata de extraer lecciones para Chile.

Palabras clave (personas en situación de calle, experiencia internacional, lecciones)

Abstract

This keynote provides some background on international experience about the topic of Homeless people and poverty, and identifies some strategies for Chilean reality. The first section discusses some conceptual and methodological issues on the magnitude and incidence of homeless people in the international context. The second section describes the profile of homeless people, in the context of developed countries, making a brief parallel with the situation in Chile. Finally, the third section discusses some of the strategies that have been used in United States and again try to draw lessons for Chile.
\end{abstract}

Key words (homeless people, international experience, lessons)

\section{Introducción}

No es muy difícil consensuar que una de las manifestaciones más extremas de la pobreza, la constituyen aquellas personas que "habitan" de manera permanente en la calle. Si bien nuestro país ha mostrado una significativa disminución en los niveles de pobreza durante la última década, hasta hace un tiempo teníamos una importante falencia por no haber recopilado mayores antecedentes sobre las personas que viven en las condiciones más precarias, como son los que viven en la calle.

En este sentido, el Catastro de personas en situación de calle elaborado por MIDEPLAN en conjunto con diversas organizaciones de la sociedad civil en Junio de 2005, es un importante aporte para dimensionar las condiciones de vida de las personas y poder de- finir propuestas de política para abordar este tema.

El catastro mencionado da cuenta que la noche del día 28 de junio, había un total de 7.254 personas que dormían en la calle o en hospederías, es decir personas en carencia de un hogar. En una alta mayoría estas personas resultaron ser hombres (85\%), más de dos tercios de las personas de la calle declararon ser solteros, y tener en promedio 46 años de edad. La permanencia promedio en la calle era de 6,6 años y la razón mayormente declarada para vivir en estas condiciones fue le hecho de tener problemas con la familia de origen y problemas económicos.

El propósito de esta nota es proporcionar algunos antecedentes sobre la experiencia internacional en este ámbito y definir algunas estrategias para la rea- 
lidad de Chile. En la primera sección se discuten algunos aspectos metodológicos y conceptuales sobre la magnitud e incidencia de las personas en situación de calle en el contexto internacional. Mientras que en la segunda sección se describe el perfil de las personas en situación de calle o sin hogar en el contexto de los países desarrollados, realizando un breve paralelo con la situación Chilena. Finalmente, en la tercera sección se discuten algunas de las estrategias que se han utilizado en los Estados Unidos y nuevamente se tratan de extraer lecciones para Chile.

\section{Aspectos metodológicos y conceptuales sobre la medición de la situación de calle}

En primer lugar, se debe considerar que la medición de la cantidad de personas en situación de calle es un tema complejo, ya que es un fenómeno que no es estático, por lo tanto debe decidirse si contabilizar a las personas que están en esta situación en un determinado momento del tiempo o bien las personas que atraviesan por esta condición por un determinado período de tiempo. En general, se distingue entre el "stock" o incidencia de la situación de calle en un momento determinado, respecto a la prevalencia, considerando a las personas que viven en estas condiciones por un determinado periodo de tiempo.

En el caso norteamericano se define a la persona sin hogar ${ }^{1}$ (Homeless) como una persona que carece de una residencia permanente, ya sea que vive en la calle, en un refugio o albergue, o en un espacio abandonado o vehículo. El reconocimiento de la inestabilidad de su situación de vivienda es el as- pecto crítico para ser considerado en esta categoría. En el estudio, realizado por el Urban Institute 2001, se distingue entre incidencia contabilizando a 842 mil personas, mientras que la prevalencia para el año en que se realizó el recuento esta llegó a 3,5 millones de personas.

Un segundo aspecto relevante para considerar, se refiere diferentes dimensiones que describen la realidad de las personas sin hogar; estas han sido desarrolladas por el Observatorio Europeo de los sin hogar.

- Dimensión física: La persona simplemente no tiene un techo bajo el cual ampararse para dormir. Este dimensión también podría ampliarse a personas que teniendo un "techo", su lugar de habitación es inadecuado.

- Dimensión Social: La persona no tiene un espacio privado para mantener sus relaciones sociales.

- Dimensión legal: La persona no tiene derecho para permanecer en el espacio que habita.

La combinación de estas tres dimensiones genera diversas categorías de situaciones "sin hogar", como puede ser la situación de un ocupante de hecho de un espacio, que si bien dispone de techo y espacio privado puede ser desalojado en cualquier momento.

Si bien pueden definirse algunos parámetros o criterios conceptuales, la mayor dificultad es de tipo práctico ya que en la mayoría de los países europeos no existen mediciones oficiales de carácter regular respecto a la situación de las personas sin hogar. Tal como se puede apreciar en la Tabla 1, existen diversas aproximaciones que tienen características comunes basadas en los conceptos descritos.

TABLA 1

ESTIMACIONES DE PERSONAS EN SITUACIÓN SIN HOGAR EN EUROPA

\begin{tabular}{|c|c|c|c|c|c|}
\hline \multicolumn{1}{|c|}{ País } & Concepto & Método & Año & $\begin{array}{c}\text { Estimación } \\
\text { de personas }\end{array}$ & $\begin{array}{c}\text { Tasa 10.000 } \\
\text { habs. }\end{array}$ \\
\hline Bélgica & Sin hogar & Conteo & 2001 & 17.000 & 17,3 \\
\hline Francia & $\begin{array}{c}\text { Sin hogar } \\
\text { (sentido estricto) }\end{array}$ & Conteo & 2003 & 46.000 & 7,7 \\
\hline Holanda & $\begin{array}{c}\text { Admisiones } \\
\text { a refugios } \\
\text { temporales }\end{array}$ & Conteo & 2001 & 8.644 & 5,3 \\
\hline Italia & Sin hogar & $\begin{array}{c}\text { Conteo } \\
\text { Expansión }\end{array}$ & 2000 & 17.000 & 3,0 \\
\hline
\end{tabular}

Review Statistics of Homeless in Europe - EOH2O03.

1 El concepto Europeo o Norteamericano de personas "sin hogar" o Homeless, es homologable al concepto de personas en situación de calle, que se utiliza en nuestro país. 
En el caso de Bélgica, "sin hogar" se refiere fundamentalmente a personas que pasan la noche en albergues, mientras que en Francia la definición es más precisa, ya que distingue entre sin hogar en "sentido estricto", es decir personas que duermen en la calle o en refugios temporales, a la vez en ese país también define a personas sin hogar en "sentido amplio" incorporando a quienes viven en residencias institucionalizadas; una distinción similar se ocupa en el caso Italiano. Finalmente, en el caso de Holanda, la contabilización se realiza en base a las solicitudes de admisión a albergues o refugios temporales; lo que incluye a personas efectivamente sin hogar, pero también a personas víctimas de crisis psicosociales como es el caso de mujeres que experimentan violencia doméstica; por lo tanto esta definición es algo más amplia. Por lo tanto, en términos conceptuales en general se distingue entre situaciones más estrictas respecto de otras más amplias. En este sentido, lo importante para un país que se inicia en este tipo de registro como es el caso de Chile, es tratar de mantener una definición coherente en el tiempo de manera de poder hacer comparaciones sobre la dinámica del fenómeno.

Un tercer aspecto metodológico que merece destacarse, es el hecho que en la mayoría de los países se realiza una contabilización en una noche determinada, ya sea por un conteo directo, o bien tomando una muestra de los albergues y residencias que ya están previamente identificados. En general, existe consenso que los conteos subestiman la realidad de las personas sin hogar. En la mayoría de los países estos conteos se concentran en las ciudades más importantes del país. Sin embargo, en el caso de Italia se realiza una expansión de los datos primarios utilizando coeficientes de expansión según el tamaño de las ciudades.

En suma, como suele suceder en la mayoría de los fenómenos sociales, la mera cuantificación del mismo no es condición suficiente para la solución de los problemas, pero sí es un importante aporte a su visibilización, y muchas veces el origen de acciones más sistemáticas como es el caso de la legislación especial que se dictó en Gran Bretaña.

\section{Características de las personas en la calle}

Tal como se puede apreciar a partir de la sección anterior, la existencia de personas en situación de calle en países de altos niveles de desarrollo, revela que este fenómeno no se asocia necesariamente a situaciones de pobreza sino más bien a problemas de exclusión y vulnerabilidad social. De hecho, la tasa de incidencia en Chile, medida como el número de personas en situación de calle cada 10.000 habitantes nos coloca en una magnitud relativa algo inferior a la de Francia, pero sobre la que se obtiene para Holanda.

Por lo tanto es interesante hacer un breve perfil de las personas sin hogar en los países desarrollados ${ }^{2}$, para comparar y sacar algunos aprendizajes que pueden ser relevantes para la realidad Chilena.

- En primer lugar, debe tenerse presente que la información disponible revela que ha existido una tendencia al aumento de las personas sin hogar en casi toda la Europa mediterránea y Anglo Sajona. Los principales grupos que han aumentado la demanda por servicios en albergues y refugios temporales son los inmigrantes, adictos y ex pacientes psiquiátricos, estos últimos debido a las políticas de desinstitucionalización de los pacientes psiquiátricos. Este perfil es similar al encontrado en el caso Norteamericano, en el cual existe una alta correlación entre personas en situación de calle y enfermedades mentales crónicas.

A pesar de que en Europa existe la percepción de que el fenómeno de los sin hogar suele asociarse a un problema de las grandes ciudades, existe diversa evidencia que muestra que ese patrón espacial puede estar cambiando, ya que hay países como Portugal y España en los cuales la mayor tasa de crecimiento de los últimos años se ha observado en ciudades intermedias. En general, no existe información sistemática respecto a los sectores rurales, pero existe la aprensión de que está largamente subestimada.

Por lo tanto, a partir de la experiencia europea es importante en el caso Chileno, revisar hasta que punto las ciudades secundarias y pueblos rurales están siendo considerados adecuadamente en los conteos. También es interesante considerar el tipo de enfermedades crónicas que enfrentan las personas en situación de calle, y si es que están consideradas con el Plan AUGE, si bien esto no les solucionaría el problema habitacional, al menos les cubriría su enfermedad.

2 La información para elaborar este perfil proviene de Edgar, Doherty y Meert (2003), y Urban Institute 2001 y 2004. 
- Las estadísticas Europeas revelan que más de dos tercios de las personas sin hogar son situaciones unipersonales, siendo predominantemente un fenómeno masculino, y personas mayores de 30 años. Desgraciadamente, la información disponible no permite definir tendencias demográficas precisas, ya que en algunos países como Bélgica y Portugal, existe un mayor crecimiento del segmento femenino debido probablemente a problemas de violencia doméstica, pero se desconocen las tendencias para el resto de los países. Algo similar ocurre con la edad, ya que países como Alemania, Francia e Italia, cerca de un 20\% de su población sin hogar tiene menos de 25 años, pero en el resto de los casos la estructura demográfica no se ha visto alterada últimamente.

A partir de este antecedente, será interesante observar para el caso Chileno en futuros conteos de las personas en situación de calle cómo evoluciona el perfil demográfico, ya que es obviamente distinto el tratar con una población que se hace cada vez más joven que con otra de mayor edad, el tipo de programas e intervenciones deberá responder a este nuevo perfil.

- Otro fenómeno de preocupación en Europa, es la creciente proporción que la población extranjera tiene entre los sin hogar que recurren a albergues o refugios temporales. En Italia y España, más del 40\% de los sin hogar son extranjeros.

Información no oficial señala que en Chile existen alrededor de 300.000 personas que han ingresado con visa de turista pero que tienen la intención de permanecer, y se trata principalmente de una migración en busca de mejores condiciones socioeconómicas. Por lo tanto, en el futuro será importante observar la nacionalidad de las personas en situación de calle.

\section{Lecciones de la estrategia Norteamericana}

En esta sección se realiza una breve reseña de algunos elementos incorporados en el trabajo de la "Alianza Nacional para Terminar con la Carencia de Hogar", que se ha implementado en los Estados Unidos bajo la responsabilidad del Department of Housing and Urban Development (HUD). Esta ini- ciativa es parte de un plan a 10 años plazo, que se inició el año 2000 y se vio reforzada por el llamado que hizo el Presidente de ese país, para "considerar como un objetivo fundamental de política, el terminar con la carencia crónica de hogar en un plazo de 10 años". Con esa fundamentación se solicitó un aporte extraordinario para el año fiscal 2003 y se creó un consejo interagencias federales para coordinar todos los esfuerzos en el tema. Por su parte, el HUD hizo suyo el propósito de eliminar la carencia crónica de hogar conjuntamente con 200 ciudades que elaboraron planes específicos en este sentido.

De acuerdo a la evaluación realizada por el Urban Institute $^{3}$, las estrategias exitosas de las ciudades para disminuir la carencia de hogar se basaron en los siguientes aspectos esenciales:

- Cambio de paradigma con un objetivo intencionado y explícito de terminar con la situación de personas en carencia crónica de hogar. Esto significa no solo tener una red interna y coordinación, sino que tener una clara decisión de política para eliminar la situación de las personas sin hogar.

- Definición de una meta precisa para eliminar la situación crónica.

- Compromiso comunitario amplio, a través de todo tipo de organizaciones.

- Liderazgo y capacidad organizacional, través de una agencia que lidera formalmente el proceso.

- Recursos suficientes más allá de los programas específicos para las personas sin hogar.

En forma complementaria, la investigación encontró que en muchos de los casos más exitosos, también contribuyó de manera significativa el acontecimiento de alguna situación o hecho puntual que detonó el interés público en el tema de los carentes de hogar.

Finalmente, la evaluación también identificó diversos elementos que, sin ser esenciales, contribuyen al éxito de la estrategia, estos son:

- Compromiso del sector privado.

- Compromiso de las autoridades locales.

- Seguimiento y monitoreo del proceso.

- Diversificación en los servicios entregados a las personas.

- Disminución de la adversidad social a proyectos de apoyo a personas en situación de carencia de hogar.

3 Estudio cualitativo en 9 ciudades de diversos estados. 
Tanto la formalidad de la estrategia reseñada, como los números absolutos de personas sin hogar para los Estados Unidos nos muestran que este problema ha alcanzado mayor relevancia y reconocimiento público, en relación a lo que sucede en el mismo ámbito para el caso Chileno.

Sin embargo, la realización del Catastro de personas en situación de calle permitió posicionar el tema de una manera concreta y sistemática. Más aún, a menos de un año de la realización de dicho estudio, MIDEPLAN dio a conocer un programa especial para incorporar a Chile Solidario a todas las personas en esta situación.

Esta iniciativa, a similitud de la estrategia Norteamericana, cuenta con una agencia que está liderando el tema como es MIDEPLAN, esto es evaluado positivamente. Del mismo modo, por ser Chile Solidario una intervención multidimensional, esta incluye no solo transferencias monetarias a las personas en situación de calle, sino también el apoyo psicosocial y la atención garantizada en los servicios públicos, particularmente en salud. Por otro lado, la iniciativa Chilena también incorpora el apoyo privado a través de instituciones sin fines de lucro quienes desarrollarán parte del componente psicosocial.

El mayor desafío que se visualiza al contrastar la situación Chilena con respecto a la extranjera, es que no se ha posicionado formalmente al nivel local como un actor relevante en el tema. Este aspecto es crítico para asegurar la sostenibilidad de los esfuerzos que se están realizando actualmente. Como es sabido, la intervención Chile Solidario se extiende por dos años y después de ese plazo, si bien hay algunos beneficios monetarios que se mantienen, no existe un proceso de acompañamiento sistemático a la persona. En este sentido, dadas las características de las personas en situación de calle, es necesario prever la mantención del apoyo personalizado e integral por un plazo más largo; esto sólo se puede lograr con una contribución más decidida del municipio. Por otro lado, también se visualiza un mayor espacio para los actores de la sociedad civil, ya no solo como prestadores de servicios específicos a las personas en situación de calle, sino también abriendo una amplia gama de intervenciones y articulaciones a través de redes mas diversas.

Un segundo desafío que también se constata para la realidad Chilena, es que el tema de las personas en situación de calle es un fenómeno todavía incipiente en la opinión pública y en el contexto de las prioridades de política. En este sentido es necesario replicar el catastro e idealmente mejorarlo metodológicamente asegurando la mejor cobertura posible en ciudades secundarias, pueblos y sectores rurales. Con esta nueva información se conseguirá mantener esta preocupación como un tema y prioridad de la política social.

\section{Bibliografía}

BURT, M; HEDDERSON J.; ZWEIG, J. et. al. (2004) Strategies for Reducing Chronic Street Homelessness. The Urban Institute, U.S. Department of Housing and Urban Development. Office of Policy Development and Research.

Conferencia Nacional de la Alianza Nacional para terminar con la Carencia de Hogar (2001).

Conferencia nacional de la alianza nacional para terminar con la carencia de hogar (2001).

Discurso del secretario Martínez dirigido a la alianza nacional para terminar con la carencia de hogar. Washington, DC.

EDGAR, B., DOHERTY, J. Y MEERT, H. (2003) Review of statistics on Homelessness in Europe. European Observatory on Homelessnes.

MIDEPLAN (2005) Habitando la calle. Catastro Nacional de Personas en Situación de Calle.

URBAN INSTITUTE. (2001) What Will It Take to End Homelessness?

U. S. DEPARTMENT OF HEALTH \& HUMAN SERVICES (2003) Ending Chronic Homelessness: trategies for Action. http://aspe.hhs.gov/hsp/homelessness/strategies03/index.htm 